\title{
Ocorrência de aflatoxina e zearalenona em alimentos destinados ao gado leiteiro na Região Norte do Estado do Paraná
}

\section{Occurrence of aflatoxins and zearalenone in feedstuffs destined to dairy cattle consumption in Northern Parana, Brazil}

\author{
Marcia Sassahara $^{1}$; Erika Kubota Yanaka ${ }^{1}$; Daisy Pontes Netto ${ }^{2 *}$
}

\section{Resumo}

\begin{abstract}
Os fungos ocorrem em uma grande variedade de alimentos, inclusive nos volumosos e concentrados destinados à alimentação animal, e podem produzir micotoxinas sob certas condições. O presente trabalho teve por objetivo avaliar a presença das micotoxinas aflatoxina e zearalenona em alimentos fornecidos a bovinos de exploração leiteira na região Norte do Estado do Paraná, Brasil, utilizando como metodologia analítica a cromatografia em camada delgada. Das 272 amostras analisadas para aflatoxinas, 37 (13,6\%) foram positivas, sendo $20(7,3 \%)$ acima do limite de $20 \mu \mathrm{g} / \mathrm{Kg}$ determinado pela ANVISA. Das 189 amostras analisadas para zearalenona, $32(16,9 \%)$ amostras foram positivas, e todas estavam acima do limite de $200 \mu \mathrm{g} / \mathrm{Kg}$ recomendado pelos veterinários da região norte do Paraná, Brasil. Foi encontrada maior contaminação por aflatoxinas em alimentos concentrados, enquanto que por zearalenona ocorreu em alimentos volumosos.
\end{abstract}

Palavras-chave: Micotoxinas, aflatoxina, zearalenona, alimentação animal, gado leiteiro.

\begin{abstract}
Molds occur in a wide range of foods and feedstuffs, including roughage and grains destined to animal consumption, and may produce mycotoxins under certain conditions. This work intended to survey the presence of mycotoxins aflatoxin and zearalenone in feedstuffs destined to dairy cattle consumption in the region of Northern in the Paraná state, Brazil, using thin layer chromatography. Of the 272 samples analyzed for aflatoxins, $37(13,6 \%)$ were positive with $20(7,3 \%)$ above the limit of $201 / \mathrm{gg} / \mathrm{Kg}$ determined by ANVISA. Of the 189 samples analyzed for zearalenone, $32(16,9 \%)$ were positive and all were above $200^{1} / 4 \mathrm{~g} / \mathrm{Kg}$, which is the recommended limit in the surveyed region. Contamination by aflatoxins occurred more in grains, while zearalenone occurred more in roughages.
\end{abstract}

Key words: Mycotoxins, aflatoxin, zearalenone, animal feedstuff, dairy cattle.

\section{Introdução}

Os fungos ocorrem em uma grande variedade de alimentos, inclusive nos volumosos e concentrados destinados à alimentação animal. A produção de micotoxinas é dependente da espécie fúngica e ocorre sob certas condições, que incluem a presença de esporos do fungo, substrato orgânico e níveis adequados de umidade, oxigênio, temperatura e acidez (MOSS, 1991; WHITLOW; HAGLER JUNIOR, 1999).

\footnotetext{
1 Aluna do Programa de Pós-graduação em Ciência Animal (área de concentração: Sanidade Animal; nível: Mestrado), Universidade Estadual de Londrina (UEL).

2 Laboratório de Toxicologia Veterinária, DMVP, CCA, UEL, Londrina, Paraná Brasil, Caixa Postal: 6001, Tel: (43) 3371-4485, e-mail:rnetto@uel.br.

*Autor para correspondência
} 
Micotoxinas são produtos metabólicos tóxicos produzidos por certas espécies de fungos, como por exemplo, Aspergillus flavus (A. flavus) e A. parasiticus, que são produtores de aflatoxinas. Embora esses fungos sejam comuns, a contaminação de alimentos depende das condições de cultivo, colheita, armazenamento e processamento (MOLIN, 1999a).

Dentre as micotoxinas que contaminam os alimentos, as mais estudadas e de maior importância econômica são as aflatoxinas, a zearalenona, os tricotecenos, a fumonisina e a ocratoxina (MOLIN, 1999b). As micotoxinas podem aumentar a incidência de doenças e diminuir a eficiência produtiva do gado. Os efeitos são decorrentes de três mecanismos primários: alteração do conteúdo nutricional, da absorção e do metabolismo; alteração na função endócrina e neuroendócrina; e supressão do sistema imune (COUNCIL FOR AGRICULTURAL SCIENCE AND TECHNOLOGY, 1989).

As aflatoxinas são as micotoxinas que mais causam preocupação, pois apresentam propriedades carcinogênicas, mutagênicas e teratogênicas, causando grandes danos à saúde humana e elevados prejuízos econômicos no rendimento de animais domésticos, tais como bovinos, ovinos, suínos, aves e coelhos (LAZZARI, 1997). A aflatoxina $\mathrm{B}_{1}\left(\mathrm{AFB}_{1}\right)$ apresenta o maior grau de toxicidade para animais, seguida das aflatoxinas $\mathrm{M}_{1}\left(\mathrm{AFM}_{1}\right), \mathrm{G}_{1}\left(\mathrm{AFG}_{1}\right), \mathrm{B}_{2}\left(\mathrm{AFB}_{2}\right)$ e $\mathrm{G}_{2}$ $\left(\mathrm{AFG}_{2}\right)$ (GOURAMA; BULLERMAN, 1995).

A produção de aflatoxinas é favorecida por temperatura e umidade elevadas, condições geralmente encontradas em regiões tropicais e subtropicais. As aflatoxinas podem ser encontradas em diferentes tipos de alimentos, como por exemplo, o milho e derivados, arroz, sorgo, amendoim, caroço de algodão e nozes, destinados tanto ao consumo humano quanto animal (RODRICKS; STOLOFF, 1977; WORLD HEALTH ORGANIZATION, 1979; PATTERSON; ROBERTS, 1980).

A zearalenona, uma lactona do ácido resorcíclico, é uma micotoxina produzida por diferentes espécies de fungos do gênero Fusarium, e apresenta efeito estrogênico em animais domésticos (HAGLER; MIROCHA; PATHRE, 1979). De ocorrência mundial, sua produção é dependente das condições climáticas sazonais, sendo mais prevalente nas estações frias e úmidas (DOKO et al., 1996; WHITLOW; HAGLER JUNIOR, 1999).

O consumo de alimentos contaminados por zearalenona determina um quadro de hiperestrogenismo nas espécies domésticas, caracterizado pela diminuição da produção de leite, repetição de cio, diminuição da taxa de concepção e até mesmo abortamento. Em muitos casos, os animais apresentam-se com aspecto saudável e escore corporal considerado normal, porém com baixa performance reprodutiva (WEAVER et al., 1986; WHITLOW; HAGLER JUNIOR, 1999).

No Brasil, as pesquisas sobre a qualidade dos alimentos destinados ao consumo animal vêm demonstrando, cada vez mais, os problemas causados pelas micotoxinas. O clima tropical e subtropical de certas regiões brasileiras é adequado ao desenvolvimento de fungos e, conseqüentemente, a produção das micotoxinas. As exigências do mercado consumidor, aliadas a esta característica climática, e o controle exercido pelas agências governamentais nacionais e internacionais, apontam para a necessidade da implementação de programas de monitoramento da qualidade dos alimentos.

A presença de micotoxinas em alimentos destinados ao consumo animal foi relatada em diferentes regiões do Brasil. Parreiras, Gomes e Brandão (1987) avaliaram a presença de $\mathrm{AFB}_{1} \mathrm{em}$ forragens e $\mathrm{AFM}_{1}$ no leite de animais, proveniente da microrregião de Viçosa, Estado de Minas Gerais, tendo sido detectada a presença de $\mathrm{AFB}_{1}$ em 9 (35\%) das 26 amostras analisadas. Purchio et al. (1988) identificaram a presença de aflatoxinas $\mathrm{AFB}_{1}$ e $\mathrm{AFG}_{1}$ em 17 (10\%) em 170 amostras de rações destinadas ao gado leiteiro, dos Estados de São Paulo e Rio Grande do Sul. Corrêa et al. (1997) avaliaram 90 amostras de rações no Estado de São Paulo, detectando a presença de $\mathrm{AFB}_{1}$ e $\mathrm{AFB}_{2}$ em 14 (15\%) amostras. 
Análises realizadas em amostras de alimentos destinados ao consumo do gado leiteiro foram positivas em 3,2\% para zearalenona (MOLIN, 1999a). Sabino, Prado e Ikejiri (1989) detectaram níveis de zearalenona que variaram entre 653 a $9.830^{1} / \mathrm{g} / \mathrm{Kg}$ em $4,5 \%$ das amostras de milho provenientes dos Estados de Santa Catarina, Minas Gerais, São Paulo, Paraná, Rio Grande do Sul e Espírito Santo. Hennigen e Dick (1995), não encontraram zearalenona em 36 amostras de milho, provenientes do Estado do Rio Grande do Sul.

A necessidade de um programa eficiente de monitoramento da qualidade e controle dos alimentos destinados ao consumo animal delineou o presente trabalho que teve como objetivo avaliar a presença de aflatoxinas e zearalenona em alimentos fornecidos a bovinos em propriedades produtoras de leite tipo $\mathrm{B}$ na região norte do Estado do Paraná, Brasil.

\section{Material e Métodos}

\section{Amostragem}

No período de julho de 2001 a novembro de 2002, em 40 propriedades produtoras de leite tipo $\mathrm{B}$, situadas em 12 municípios da região norte do Estado do Paraná, foram coletadas 272 amostras para análise de aflatoxinas e 189 amostras para análise de zearalenona, conforme apresentado na Tabela 1. As amostras eram formadas por alimentos volumosos (silagens) e concentrados (grãos e rações comerciais ou preparadas nas propriedades) fornecidos como alimentação ao gado leiteiro.

As amostras de silagem foram coletadas em nove pontos distintos do silo: três pontos do terço superior do silo, três pontos do terço médio e três pontos do terço inferior. O material foi então homogeneizado e a análise efetuada em uma alíquota de $100 \mathrm{~g}$, representativa da face exposta do silo. As amostras foram acondicionadas em embalagens plásticas, transportadas a $4^{\circ} \mathrm{C}$ e armazenadas a $-20^{\circ} \mathrm{C}$ até seu processamento.
Tabela 1. Número de amostras de alimentos destinados ao consumo animal, coletadas em propriedades produtoras de leite tipo B, de 12 municípios da região norte do Estado do Paraná, para análise de aflatoxinas e zearalenona pelo método de cromatografia em camada delgada, durante o período de julho de 2001 a novembro de 2002

\begin{tabular}{cccc}
\hline \multirow{2}{*}{ Município } & \multirow{2}{*}{$\begin{array}{c}\text { Número de } \\
\text { propriedades }\end{array}$} & \multicolumn{2}{c}{$\begin{array}{c}\text { Amostras coletadas para } \\
\text { análise de }\end{array}$} \\
\cline { 3 - 4 } & & aflatoxinas & zearalenona \\
\hline Apucarana & 8 & 36 & 20 \\
Arapongas & 3 & 7 & 0 \\
Cambé & 1 & 21 & 15 \\
Cambira & 5 & 46 & 30 \\
Floresta & 4 & 14 & 14 \\
Jandaia do Sul & 2 & 5 & 0 \\
Mandaguari & 5 & 10 & 0 \\
Marialva & 2 & 25 & 25 \\
Quinta do Sol & 1 & 8 & 8 \\
Rio Bom & 7 & 75 & 60 \\
Rolândia & 1 & 17 & 9 \\
Sarandi & 1 & 8 & 8 \\
\hline Total & 40 & 272 & 189 \\
\hline
\end{tabular}

A coleta de amostras de alimentos concentrados foi realizada nos galpões onde os alimentos eram armazenados, ou em silos graneleiros. No caso de concentrados armazenados em sacos, foram coletadas amostras aleatórias dos sacos destinados ao próximo arraçoamento dos animais. No caso de silos graneleiros, as amostras foram coletadas pelo método de "fluxo movente", com um recipiente passado a cada 2 minutos através de um fluxo de grãos por um período de 10 minutos. Em ambos os casos, as sub-amostras foram misturadas e então retirada uma alíquota de $100 \mathrm{~g}$, que foi acondicionada em embalagem de papel e mantida à temperatura ambiente até seu processamento.

Todas as rações preparadas foram constituídas de grãos e farelos provenientes de cooperativas situadas nos municípios de Londrina e Apucarana, Estado do Paraná, Brasil e todas as rações comerciais, de diferentes marcas, também foram provenientes de cooperativas da mesma região. Em ambos os casos, os principais ingredientes das rações foram o milho e a soja. 


\section{Metodologia analítica}

As amostras foram analisadas utilizando-se a metodologia de cromatografia em camada delgada (CCD) descrita por Soares e Rodriguez-Amaya (1989) e adaptada pelo Instituto Adolfo Lutz/São Paulo (1996).

Os padrões utilizados para a técnica de CCD foram $\mathrm{AFB}_{1}, \mathrm{AFB}_{2}, \mathrm{AFG}_{1}, \mathrm{AFG}_{2}$ e zearalenona (Sigma Inc. - EUA), nas concentrações de 2,55, 2,62, $2,45,4,55$ e $68 \mu \mathrm{g} / \mathrm{ml}$ respectivamente, preparados conforme metodologia da Association of Official Analytical Chemists (AOAC) também adaptada pelo Instituto Adolfo Lutz (1996).

Os limites de detecção do método são 2 e $55 \mu \mathrm{g}$ e os limites de determinação, 4 e $165 \mu \mathrm{g}$ para aflatoxina e zearalenona, respectivamente. A confirmação para a presença de aflatoxinas foi realizada pela prova de reação química com o ácido trifluoroacético, de acordo com o método também descrito pelo Instituto Adolfo Lutz (1996).

\section{Resultados e Discussão}

O resultado das análises para a detecção de aflatoxinas nos alimentos destinados ao consumo do gado leiteiro pode ser observado na Tabela 2. A maior porcentagem de contaminação por essas toxinas foi observada nas rações, tanto comerciais quanto preparadas.

O limite máximo permitido de aflatoxinas nos grãos varia de acordo com a legislação de cada país. Os limites permitidos no Brasil para alimentos destinados ao consumo humano são de $20 \mu \mathrm{g} / \mathrm{Kg}$ de $\mathrm{AFB}_{1}+\mathrm{AFB}_{2}+\mathrm{AFG}_{1}+\mathrm{AFG}_{2}$ para milho em grão (inteiro, partido, amassado e moído), farinhas ou sêmolas de milho, amendoim e derivados, e $0,5 \mu \mathrm{g} / \mathrm{l}$ de $\mathrm{AFM}_{1}$ em leite fluido (AGÊNCIA NACIONAL DE VIGILÂNCIA SANITÁRIA, 2002). O Ministério da Agricultura, Pecuária e Abastecimento do Brasil adota o limite de $50 \mu \mathrm{g} / \mathrm{Kg}$ para alimentos destinados ao consumo animal (ingestão direta ou matéria-prima de rações) (BRASIL, 2002).
Tabela 2. Número e tipo de amostras coletadas, de alimentos destinados ao consumo animal, em propriedades produtoras de leite tipo B, de 12 municípios da região norte do Estado do Paraná, no período de julho de 2001 a novembro de 2002 e os resultados obtidos nas análises para a detecção de aflatoxinas $\mathrm{B}_{1}+\mathrm{B}_{2}+\mathrm{G}_{1}+\mathrm{G}_{2}$ realizadas pelo método de cromatografia em camada delgada

\begin{tabular}{|c|c|c|c|c|c|}
\hline \multirow{2}{*}{ Tipo de amostra } & \multicolumn{2}{|c|}{ Positivo } & \multicolumn{2}{|c|}{ Negativo } & \multirow{2}{*}{ Total } \\
\hline & $\mathrm{N}^{\mathrm{o}}$ & $\%$ & $\mathrm{~N}^{\mathrm{o}}$ & $\%$ & \\
\hline Ração comercial & 17 & 20 & 68 & 80 & 85 \\
\hline Ração preparada & 12 & 36 & 21 & 64 & 33 \\
\hline Silagem de milho & 1 & 0,9 & 105 & 99,1 & 106 \\
\hline Silagem de sorgo & 0 & 0 & 12 & 100 & 12 \\
\hline Silagem de milho e sorgo & 0 & 0 & 5 & 100 & 5 \\
\hline Caroço de algodão & 0 & 0 & 12 & 100 & 12 \\
\hline Farelo de soja & 1 & 7 & 12 & 93 & 13 \\
\hline Farelo de milho & 5 & 100 & 0 & 0 & 5 \\
\hline Gérmen de trigo & 1 & 100 & 0 & 0 & 1 \\
\hline Total & 37 & 13,6 & 235 & 86,3 & 272 \\
\hline
\end{tabular}

A legislação comum aos países integrantes do MERCOSUL segue os limites determinados pela ANVISA, onde o limite máximo permitido de aflatoxinas é de $20 \mu \mathrm{g} / \mathrm{Kg}$ de alimento.

Na Tabela 3 observam-se os níveis de cada aflatoxina encontrada nas 17 amostras de ração comercial positivas, e a concentração total de aflatoxinas em cada amostra.

Das 85 amostras de ração comercial analisadas, em 17 (20\%) foi possível detectar a presença de aflatoxinas. Este resultado é superior ao encontrado por Purchio et al. (1987), que relataram uma contaminação por aflatoxina total em 17 (10\%) das 170 amostras de ração comercial avaliadas. Porém, apenas $3(3 \%)$ e $6(7 \%)$ amostras estavam contaminadas por $\mathrm{AFB}_{1}$ e $\mathrm{AFB}_{2}$, respectivamente, diferindo dos resultados obtidos por Corrêa et al. (1997), que encontraram 15\% das 96 amostras de ração analisadas contendo essas toxinas, e Parreiras, Gomes e Brandão (1987) que analisaram 26 amostras de ração e encontraram 9 (35\%) positivas. 
Tabela 3. Níveis detectados de aflatoxinas, em 17 amostras de rações comerciais destinadas ao consumo animal, coletadas em propriedades produtoras de leite tipo B, de 12 municípios da região norte do Estado do Paraná, analisadas pelo método de cromatografia em camada delgada, durante o período de julho de 2001 a novembro de $2002(\mathrm{em} \mu \mathrm{g} / \mathrm{Kg})$

\begin{tabular}{cccccc}
\hline $\mathrm{N}^{0}$ da amostra & $\mathrm{AFB}_{1}$ & $\mathrm{AFB}_{2}$ & $\mathrm{AFG}_{1}$ & $\mathrm{AFG}_{2}$ & $\begin{array}{c}\text { Aflatoxina } \\
\text { total }\end{array}$ \\
\hline 25 & $(-)$ & $(-)$ & 22,40 & $(-)$ & 22,40 \\
38 & $(-)$ & $(-)$ & $(-)$ & 6,75 & 6,75 \\
45 & $(-)$ & $(-)$ & 5,60 & $(-)$ & 5,60 \\
53 & $(-)$ & $(-)$ & 5,60 & $(-)$ & 5,60 \\
90 & $(-)$ & $(-)$ & 7,84 & 14,56 & 22,40 \\
112 & $(-)$ & 8,38 & $(-)$ & $(-)$ & 8,38 \\
116 & $(-)$ & 8,38 & 15,68 & $(-)$ & 24,06 \\
129 & $(-)$ & 8,38 & $(-)$ & 29,12 & 37,50 \\
130 & $(-)$ & $(-)$ & $(-)$ & 29,12 & 29,12 \\
136 & $(-)$ & $(-)$ & 7,84 & $(-)$ & 7,84 \\
138 & 8,16 & 8,38 & $(-)$ & 14,56 & 31,10 \\
140 & 8,16 & 16,76 & $(-)$ & $(-)$ & 24,92 \\
143 & 8,16 & 8,38 & 7,84 & $(-)$ & 24,38 \\
170 & $(-)$ & $(-)$ & $(-)$ & 14,56 & 14,56 \\
190 & $(-)$ & $(-)$ & 7,84 & $(-)$ & 7,84 \\
196 & $(-)$ & $(-)$ & 7,84 & $(-)$ & 7,84 \\
250 & $(-)$ & $(-)$ & 7,84 & $(-)$ & 7,84 \\
\hline
\end{tabular}

(-): Negativo para nível de detecção de $2 \mu \mathrm{g}$

$\mathrm{O}$ trecho destacado representa positividade acima do limite de $20 \mu \mathrm{g} / \mathrm{Kg}$.

Das 17 amostras positivas para aflatoxina, 8 (47\%) apresentaram-se acima dos níveis de $20 \mu \mathrm{g} /$ Kg permitidos pela ANVISA, e nenhuma amostra acima de $50 \mu \mathrm{g} / \mathrm{Kg}$. Os resultados encontrados foram diferentes dos relatados por outros autores. Corrêa et al. (1997) relatam apenas duas amostras acima de $50 \mu \mathrm{g} / \mathrm{Kg}$, porém alcançando níveis máximos de até $287 \mu \mathrm{g} / \mathrm{Kg}$ de $\mathrm{AFB}_{1}$, enquanto apenas uma das amostras de Purchio et al. (1988) estava abaixo de $20 \mu \mathrm{g} / \mathrm{Kg}$, quatro abaixo de $50 \mu \mathrm{g} / \mathrm{Kg}$, e as 12 restantes acima deste limite, atingindo valores máximos de $200 \mu \mathrm{g} / \mathrm{Kg}$ para $\mathrm{AFB}_{1}$. Parreiras, Gomes e Brandão (1987) relataram que nove amostras positivas apresentavam níveis de $\mathrm{AFB}_{1}$ entre 7,7 e $30 \mu \mathrm{g} / \mathrm{Kg}$, sendo quatro abaixo do limite de $20 \mu \mathrm{g} / \mathrm{Kg}$.

Embora as rações comerciais utilizadas nas propriedades estudadas neste trabalho sejam comercializadas com garantia do fabricante de níveis de aflatoxinas controlados (entre 20 e $50 \mu \mathrm{g} / \mathrm{Kg}$, variando de acordo com a marca), o armazenamento inadequado das rações nas propriedades pode favorecer o crescimento fúngico e uma subseqüente contaminação e aumento da concentração de aflatoxinas.

Das quatro propriedades que utilizavam silos graneleiros para a armazenagem de ração comercial, apenas uma realizava a limpeza do interior do silo entre os novos carregamentos de rações adquiridas. Nenhuma das amostras de ração comercial dessa propriedade apresentou contaminação por aflatoxina.

A freqüência de rações preparadas na propriedade, contaminadas por aflatoxinas, foi maior (36\%), do que as rações comerciais. Todas as propriedades preparavam as rações em quantidade suficiente para, no mínimo, uma semana, as quais eram mantidas em embalagens reutilizadas, sem a prévia limpeza entre cada reutilização.

Das 12 amostras de rações preparadas analisadas, observou-se que oito (67\%) apresentam-se acima do limite de $20 \mu \mathrm{g} / \mathrm{Kg}$, atingindo níveis máximos de $29,04 \mu \mathrm{g} / \mathrm{Kg}$ de $\mathrm{AFB}_{1}$ e $71,08 \mu \mathrm{g} / \mathrm{Kg}$ de aflatoxina total (Tabela 4).

Tabela 4. Níveis detectados de aflatoxinas, em 12 amostras de rações preparadas destinadas ao consumo animal, coletadas em propriedades produtoras de leite tipo B, de 12 municípios da região norte do Estado do Paraná, analisadas pelo método de cromatografia em camada delgada, durante o período de julho de 2001 a novembro de 2002 (em $\mu \mathrm{g} / \mathrm{Kg}$ )

\begin{tabular}{cccccc}
\hline $\mathrm{N}^{0}$ da amostra & $\mathrm{AFB}_{1}$ & $\mathrm{AFB}_{2}$ & $\mathrm{AFG}_{1}$ & $\mathrm{AFG}_{2}$ & $\begin{array}{c}\text { Aflatoxinas } \\
\text { totais }\end{array}$ \\
\hline 1 & $(-)$ & $(-)$ & $(-)$ & 27,00 & 27,00 \\
4 & 7,26 & 7,00 & 5,60 & 6,75 & 26,61 \\
20 & $(-)$ & 7,00 & $(-)$ & $(-)$ & 7,00 \\
30 & 29,04 & 42,04 & $(-)$ & $(-)$ & 71,08 \\
41 & $(-)$ & $(-)$ & $(-)$ & 6,75 & 6,75 \\
43 & 7,26 & 14,00 & $(-)$ & $(-)$ & 21,26 \\
49 & 7,26 & 7,00 & 5,60 & 6,75 & 26,61 \\
64 & 14,52 & $(-)$ & $(-)$ & $(-)$ & 14,52 \\
65 & 7,26 & $(-)$ & $(-)$ & 13,50 & 20,76 \\
110 & 8,16 & 25,15 & $(-)$ & $(-)$ & 33,31 \\
114 & 8,16 & 16,76 & $(-)$ & $(-)$ & 24,92 \\
187 & 8,16 & 8,38 & $(-)$ & $(-)$ & 16,54 \\
\hline
\end{tabular}

(-): Negativo para nível de detecção de $2 \mu \mathrm{g}$

$\mathrm{O}$ trecho destacado representa positividade acima do limite de $20 \mu \mathrm{g} / \mathrm{Kg}$. 
O preparo de rações nas propriedades pode aumentar o risco de intoxicação dos animais, pois são utilizados grãos de diferentes procedências e sem garantia de controle dos níveis de micotoxinas, ao contrário do que ocorre com as rações comerciais.

Outro fator que pode contribuir para o aumento da contaminação é a presença de roedores nos galpões onde as rações são armazenadas. A violação das embalagens pelos roedores facilita o aumento de umidade, além do fato de esses animais atuarem como carreadores dos fungos para o interior da embalagem. Em quatro propriedades foi observada a presença de roedores entre os sacos de ração durante a coleta das amostras, enquanto em outras sete propriedades pôde-se observar marcas de infiltração nas paredes e telhado dos galpões, o que favorece o aumento da umidade no interior dos galpões.

Uma amostra de silagem de milho e uma amostra de farelo de soja apresentaram-se positivas para aflatoxinas (Tabela 5). Uma única amostra de farelo de trigo foi analisada, não sendo possível concluir a importância da contaminação deste tipo de grão.

Tabela 5. Níveis detectados de aflatoxinas, em amostras de silagem, farelo de soja, farelo de milho e gérmen de trigo coletadas em propriedades produtoras de leite tipo B, de 12 municípios da região norte do Estado do Paraná, analisadas pelo método de cromatografia em camada delgada, durante o período de julho de 2001 a novembro de 2002 (em $\mu \mathrm{g} / \mathrm{Kg}$ )

\begin{tabular}{cccccc}
\hline $\mathrm{N}^{\mathrm{o}}$ da amostra & $\mathrm{AFB}_{1}$ & $\mathrm{AFB}_{2}$ & $\mathrm{AFG}_{1}$ & $\mathrm{AFG}_{2}$ & $\begin{array}{c}\text { Aflatoxina } \\
\text { total }\end{array}$ \\
\hline 240 (silagem de milho) & $(-)$ & 8,38 & $(-)$ & 14,56 & 22,94 \\
123 (farelo de soja) & 8,16 & 25,15 & $(-)$ & $(-)$ & 33,31 \\
33 (farelo de milho) & $(-)$ & $(-)$ & 22,40 & $(-)$ & 22,40 \\
86 (farelo de milho) & 8,16 & 8,38 & 31,36 & 14,56 & 62,46 \\
101 (farelo de milho) & $(-)$ & $(-)$ & 7,84 & $(-)$ & 7,84 \\
134 (farelo de milho) & $(-)$ & 8,38 & 7,84 & $(-)$ & 16,22 \\
194 (farelo de milho) & $(-)$ & $(-)$ & 7,84 & $(-)$ & 7,84 \\
74 (gérmen de trigo) & 7,26 & 7,00 & 5,60 & $(-)$ & 19,86 \\
\hline
\end{tabular}

(-): Negativo para nível de detecção de $2 \mu \mathrm{g}$

$\mathrm{O}$ trecho destacado representa positividade acima do limite de $20 \mu \mathrm{g} / \mathrm{Kg}$.

Todas as amostras de farelo de milho analisadas foram positivas para aflatoxinas. Uma justificativa para a positividade de tais amostras é a utilização de grãos de qualidade inferior para a produção de farelo, numa tentativa de redução de custos. Um outro fator que predispõe a contaminação deste tipo de derivado é o seu processamento, pois danos mecânicos ou a quebra dos grãos alteram ou eliminam a cutícula do grão de milho, que serve de barreira natural contra a contaminação fúngica. Danos mecânicos de grãos, sementes e plantas favorecem a absorção de umidade e facilitam a invasão e a penetração de fungos no interior altamente nutritivo destes substratos, levando ao rápido desenvolvimento dos fungos e conseqüentemente o aumento na concentração de toxinas (SCUSSEL, 1998).

Para os seres humanos além do risco de ingestão direta de grãos contaminados, também há a possibilidade da contaminação por ingestão de produtos de origem animal. $\mathrm{A} \mathrm{AFB}_{1}$ ingerida pelos animais pode ser biotransformada em $\mathrm{AFM}_{1}$, que é excretada no leite e ovos. A AFM também apresenta atividade carcinogênica e mutagênica (PIVA et al., 1987; EL-NEZAMI et al., 1995). Estudos determinaram que a quantidade de $\mathrm{AFM}_{1}$ excretada no leite varia entre 1 a $3 \%$ da quantidade de $\mathrm{AFB}_{1}$ ingerida (NABNEY et al., 1967; MASRI; GARCIA; PAGE, 1969; POLAN; HAYES; CAMPBELL, 1974), embora valores de até 6\% tenham sido relatados por Pitet (1998).

A $\mathrm{AFM}_{1}$ é estável em leite cru e produtos derivados, e nãoé afetada por pasteurização e tratamento por UHT (ultra-high temperature) (GELOSA; BUZZETTI, 1994; GALVANO; GALORARO; GALVANO, 1996), bem como pelo processamento do leite em certos tipos de queijo e iogurte (STUBBLEFIELD; SHANNON, 1974; VAN EGMOND et al., 1977). Portanto, as principais estratégias de controle de risco de ingestão de aflatoxinas são a determinação de limites destas substâncias nos alimentos destinados a consumo humano, limitação para o consumo animal, para que não ocorra contaminação de produtos de origem animal, e programas de monitoramento para diminuir o risco de exposição.

O resultado das análises para detecção de zearalenona é descrito na Tabela 6. Observa-se que 
duas das 65 amostras de rações comerciais estavam contaminadas com esta toxina, ao contrário do que ocorreu com a aflatoxina.

Tabela 6. Número e tipo de amostras coletadas, de alimentos destinados ao consumo animal, em propriedades produtoras de leite tipo $\mathrm{B}$, de 12 municípios da região norte do Estado do Paraná, no período de julho de 2001 a novembro de 2002 e os resultados obtidos nas análises para detecção de zearalenona realizadas pelo método de cromatografia em camada delgada

\begin{tabular}{|c|c|c|c|c|c|}
\hline \multirow{2}{*}{ Tipo de amostra } & \multicolumn{2}{|c|}{ Positivo } & \multicolumn{2}{|c|}{ Negativo } & \multirow{2}{*}{ Total } \\
\hline & $\mathrm{N}^{\mathrm{o}}$ & $\%$ & $\mathrm{~N}^{\mathrm{o}}$ & $\%$ & \\
\hline Ração comercial & 2 & 3 & 63 & 97 & 65 \\
\hline Ração preparada & 0 & 0 & 18 & 100 & 18 \\
\hline Silagem de milho & 24 & 34 & 46 & 66 & 70 \\
\hline Silagem de sorgo & 4 & 44 & 5 & 56 & 9 \\
\hline Silagem de milho e sorgo & 1 & 25 & 3 & 75 & 4 \\
\hline Caroço de algodão & 0 & 0 & 11 & 100 & 11 \\
\hline Farelo de soja & 1 & 12 & 7 & 88 & 8 \\
\hline Farelo de milho & 1 & 25 & 3 & 75 & 4 \\
\hline Total & 33 & 17,46 & 156 & 82,54 & 189 \\
\hline
\end{tabular}

No Brasil, os limites permitidos para zearalenona ainda não foram estabelecidos. No Uruguai são permitidos limites máximos de $200 \mu \mathrm{g} / \mathrm{Kg}$ para milho e cevada; na Áustria, os limites permitidos para rações de suínos matrizes são $50 \mu \mathrm{g} / \mathrm{Kg}$; na França, $200 \mu \mathrm{g} / \mathrm{Kg}$ para cereais em geral e óleos vegetais; na Itália, $100 \mu \mathrm{g} / \mathrm{Kg}$ para cereais (FOOD AND AGRICULTURE ORGANIZATION, 1996).

O nível de contaminação por zearalenona presente nas duas amostras de ração contaminadas foi de $220,67 \mu \mathrm{g} / \mathrm{Kg}$. Este nível é superior aos limites recomendado para os alimentos destinados ao gado leiteiro pela legislação uruguaia, que é utilizada pelos médicos veterinários da região em que as amostras foram coletadas.

Do mesmo modo, todas as amostras de silagens e farelos positivas para zearalenona apresentaram níveis superiores a $200 \mu \mathrm{g} / \mathrm{Kg}$, conforme se observa na Tabela 7.
Tabela 7. Níveis detectados de zearalenona em amostras de silagens de milho, sorgo e milho+sorgo e farelos de soja e milho coletadas em propriedades produtoras de leite tipo B, de 12 municípios da região norte do Estado do Paraná, analisadas pelo método de cromatografia em camada delgada, durante o período de julho de 2001 a novembro de $2002(\mathrm{em} \mu \mathrm{g} / \mathrm{Kg}$ )

\begin{tabular}{cc}
\hline $\mathrm{N}^{\mathrm{o}}$ de amostras & Zearalenona \\
\hline 01 (silagem de milho) & 596,60 \\
07 (silagem de milho) & 441,34 \\
02 (silagem de milho) & 220,67 \\
04 (silagem de milho) & 882,68 \\
08 (silagem de milho) & 662,01 \\
03 (silagem de milho) & 288,73 \\
01 (silagem de sorgo) & 441,34 \\
01 (silagem de sorgo) & 577,47 \\
01 (silagem de sorgo) & 662,01 \\
01 (silagem de milho+sorgo) & 662,01 \\
01 (farelo de soja) & 220,67 \\
01 (farelo de milho) & 441,34 \\
\hline
\end{tabular}

Os resultados encontrados mostram que, para ambas as micotoxinas analisadas, a contaminação na região estudada foi diferente da descrita por Parreiras, Gomes e Brandão (1987), Purchio et al. (1988), Sabino, Prado e Ikejiri (1989), Hennigen e Dick (1995) e Corrêa et al. (1997). A metodologia analítica utilizada por esses autores foi a cromatografia líquida de alta eficiência (CLAE). A CLAE é mais sensível que a cromatografia em camada delgada, o que justificaria a diferença dos resultados obtidos. As diferenças climáticas e de temperatura entre as regiões estudadas, além da forma e tipo de armazenamento também podem justificar os resultados.

Mesmo dentro de uma única região, pode-se observar diferença entre resultados obtidos em diferentes períodos do ano, devido a alterações sazonais ou efeitos climáticos.

\section{Conclusões}

Embora todos os tipos de alimentação animal avaliadas apresentaram contaminação, por ambas as micotoxinas analisadas, a contaminação por aflatoxinas foi maior em alimentos concentrados (rações comerciais e preparadas e grãos), enquanto 
que por zearalenona foi maior em alimentos volumosos (silagens).

Embora a legislação brasileira não estabeleça limites de tolerância para a zearalenona, todas as silagens e substratos utilizados na composição de rações e alimentos para uso animal positivos para zearalenona apresentaram níveis acima do permitido pelos países que têm controle sobre este tipo de micotoxina e que é de 50 a $200 \mu \mathrm{g} / \mathrm{Kg}$.

As rações comerciais, mesmo com níveis de garantia dos fabricantes, estão sujeitas à contaminação e recontaminação por fungos, e conseqüentemente pelas micotoxinas, ocasionado, principalmente, pelo armazenamento inadequado dos sacos de ração dentro das propriedades, sendo necessário um melhor monitoramento das condições de armazenagem.

A estrutura física dos locais de armazenagem e a presença de roedores são fatores que contribuem para a contaminação.

As rações preparadas nas propriedades apresentaram uma porcentagem maior de amostras contaminadas por aflatoxina, com níveis mais elevados que dos outros tipos de amostra analisados, possivelmente, pela má qualidade do substrato utilizado ou por contaminação dos equipamentos para mistura dos ingredientes destes alimentos.

\section{Referências}

AGÊNCIA NACIONAL DE VIGILÂNCIA SANITÁRIA. Resolução - RDC $n^{\circ}$ 274. Regulamento técnico MERCOSUL sobre limites máximos de aflatoxinas admissíveis no leite, no amendoim, no milho. Disponível em: < http://www.anvisa.gov.br/legis/resol/2002/ 274_02rdc.htm>. Acesso em: 3 dez. 2002.

BRASIL. Portaria ${ }^{\circ} 07$ de 09 de novembro de 1988. Baixa os Padrões mínimos de matéria prima detinada à alimentação animal. Disponível em: <http:// www.agricultura.gov.br/dfpa/html/port07.htm>. Acesso em: 3 dez. 2002.

CORREAA, B.; GALHARDO, M.; COSTA, E.; SABINO, M. Distribution of molds and aflatoxins in dairy cattle feeds and raw milk. Revista de Microbiologia, São Paulo, v. 28, n.4, p. 279-283, 1997.
COUNCIL FOR AGRICULTURAL SCIENCE AND TECHNOLOGY. Mycotoxins: economic and health risks. Ames, 1989. (Task Force Report n ${ }^{\circ} 116$ ).

DOKO, M. B.; BROWN, N.; SYDENHAM, E. W. MPUCHANE, S.; SIAME, B. A. Natural co-occurrence of fumonisins and zearalenone in cereals and cereal-based foods from Eastern and Southern Africa. Journal of Agricultural and Food Chemistry, Easton, v. 44, n.10, p. 3240-3243, 1996.

EL-NEZAMI,H.S.;NICOLETTI, G.;NEAL, G.E.;DONOHUE, D. C.; AHOKAS, J. T. Aflatoxin $\mathrm{M}_{1}$ in human breast milk samples from Victoria, Australia and Thailand. Food and Chemical Toxicology, Elmsford, v.33, n.3, p.173-179, 1995.

FOOD AND AGRICULTURE ORGANIZATION OF THE UNITED NATIONS. Worldwide Regulations for Mycotoxins, 1995. A Compendium, Roma, n. 64, p.9-20, 1996.

GALVANO, F:; GALORARO, V.; GALVANO, G. Occurrence and stability of aflatoxin $\mathrm{M}_{1}$ in milk and milk products: a worldwide review. Journal of Food Protection, Des Moines, v. 59, n.10, p. 1076-1090, 1996.

GELOSA, M.; BUZZETTI, Y. Effetto del trattamento termico del latte sul contenuto di aflatoxina $\mathrm{M}_{1}$. Sciencia Alim., v.23, p.115-117, 1994.

GOURAMA, N.; BULLERMAN, L. B. Aspergillus flavus and Aspergillus parasiticus: Aflatoxigenic fungi of concern in foods and feeds: a review. Journal of Food Protection, Des Moines, v.58, n.12, p.1395-1404, 1995.

HAGLER, W. M.; MIROCHA, C. J. PATHRE, S. V. Identification of the naturally occurring isomer of zearalenol produced by Fusarium roseum 'Gibbosum' in rice culture. Applied and Environmental Microbiology, Washington, v.37, n.5, p.849-853, 1979.

HENNIGEN, M. R.; DICK, T. Incidence and abundance of mycotoxins in maize in Rio Grande do Sul, Brazil. Food Additives and Contaminants, London, v.12, n.5, p.677-681, 1995.

INSTITUTO ADOLFO LUTZ. Métodos para determinação simultânea de aflatoxina $B$ e G, ocratoxina A, zearalenona e esterigmatocistina. São Paulo, 1996.

LAZZARI, F. A. Umidade, fungos e micotoxinas na qualidade de sementes, grãos e rações. 2.ed. Curitiba: Ed. do Autor, 1997.

MASRI, M. S.; GARCIA, V. C.; PAGE, J. R. The aflatoxin M content of milk from cows fed known amounts of aflatoxin. Veterinary Record, London, v.84, n.6, p.146-147, 1969.

MOLIN, R. Ocorrência de micotoxinas em estágios fenológicos próximos da colheita de milho. In: MOLIN, R; VALENTINI, M. L. Simpósio Sobre Micotoxinas em Grãos. São Paulo: Fundação Cargil, 1999a. p.57-80 
MOLIN, R. Micotoxinas em grãos: contaminantes naturais de alimentos e rações. In: MOLIN, R; VALENTINI, M. L. Simpósio Sobre Micotoxinas em Grãos. São Paulo: Fundação Cargil, 1999b.

MOSS, M. O. The environmental factors controlling mycotoxin formation. In: SMITH, J. E.; HENDERSON, R. S. Mycot. and Animal Foods. Boca Raton: CRC Press, 1991.

NABNEY, J.; BURBAGE, M. B.; ALLCROFT, R.; LEWIS, G. Metabolism of aflatoxina in the lactating ewe. Food and Cosmetics Toxicology, Elmsford, v.5, p.11, 1967.

PARREIRAS, J. F. M.; GOMES, J. C.; BRANDÃO, S. C Ocorrência de aflatoxinas $\mathrm{M}_{1}$ e $\mathrm{B}_{1}$ em leite e forragens na microrregião de Viçosa - MG. Arquivos de Biologia e Tecnologia, Curitiba, v. 30, n. 21, p 253-265, 1987.

PATTERSON, D. S. P.; ROBERTS, B. A. Aflatoxin B in dairy concentrates and other animal feedstuffs. Veterinary Record, London, v. 107, n.11, p. 249-252, 1980.

PITET, A. Natural occurrence of mycotoxins in foods and feeds: an updated review. Revue de Medecine Veterinaire., Toulouse, v.149, n.6, p. 479-492, 1998.

PIVA, G.; PIETRI, A.; GALLAZZI, L.; CURTO, O. Aflatoxin M1 occurrence in dairy products marketed in Italy. Food Additives and Contaminants, London, v.5, n.2, p.133-139, 1987.

POLAN, C. E.; HAYES, J. R.; CAMPBELL, T. C. Consumption and fate of aflatoxin $\mathrm{B}_{1}$ by lactating cows. Journal of Agricultural and Food Chemistry, Easton, v. 22, no 4, p. 635-638, 1974.

PURCHIO, A.; GAMBALE, W.; PAULA, C. R.; BARBIERI, W. Micotoxinas (aflatoxinas, patulina, ocratoxina A e esterigmatocistina) e correspondentes fungos micotoxigênicos em rações destinadas ao gado leiteiro. Revista de Microbiologia, São Paulo, v. 19, n2, p.172-176, 1988.

RODRICKS, J. V.; STOLOFF, L. Aflatoxin residues from contaminated feed in edible tissues of food-producing animals. In: RODRICKS, J. V; HESSELTINE, C. W.; MEHLMAN, M. A. Mycotoxins in Human and Animal Health. Park Forest South: Pathotox Publ Inc., 1977. p. 67-69.
SABINO, M.; PRADO, G.; IKEJIRI, E. Natural occurrence of aflatoxins and zearalenone in maize in Brazil. Food Additives and Contaminants, London, v. 6, n. 3, p 327$331,1989$.

SCUSSEL, V. M. Micotoxinas em alimentos. Florianópolis: Insular, 1998.

SOARES, L. M. V.; RODRIGUEZ-AMAYA, D. B. Survey of aflatoxins, ochratoxin $A$, zearalenone and sterigmaticystin in some Brazilian foods by using multitoxin thin-layer chromatographic method. Journal AOAC International, Arlington, v.72, n.1, p.22-26, 1989.

STUBBLEFIELD, R. D.; SHANNON, G. M. Aflatoxin M, analysis in dairy products and distribution in dairy foods made from artificially contaminated milk. Journal AOAC International, Arlington, v.57, n.4, p.847-851, 1974.

VANEGMOND, H. P.; PAULSCH, W. E.; VERINGA, H. A.; SCHULLER, P. L. The effect of processing on the aflatoxina $\mathrm{M}_{1}$ content of milk and milk products. Archives De L' Institut Pasteur De Tunis, Tunis, v.54, p.381, 1977.

WEAVER, G. A.; KURTZ, H. J.; BEHRENS, J. C.; ROBINSON, T. S.; SEGUIN, B.E.; BATES, F.Y.; MIROCHA, C.J. Effect of zearalenone on the fertility of virgins dairy heifers. American Journal of Veterinary Research, Chicago, v.47, n. 6, p.1395-1397, 1986.

WHITLOW, L. W.; HAGLER JUNIOR, W. M. Mycotoxins in dairy cattle. In: MOLIN, R; VALENTINI, M. L. Simpósio Sobre Micotoxinas em Grãos. São Paulo: Fundação Cargil, 1999. p. 151-181.

WORLD HEALTH ORGANIZATION. Mycotoxins. Geneva, 1979. (Environmental Health Criteria, 11) 
